\title{
Phenotypic changes in mycobacteria grown in oxygen-limited conditions
}

\author{
JANE GILLESPIE, L. L. BARTON* and E. W. RYPKAT \\ Department of Biology, University of New Mexico, Albuquerque, NM 87131 and tSection of Microbiology, \\ Lovelace Medical Center, Albuquerque, NM 87108 USA
}

\begin{abstract}
Summary. Laboratory strains of Mycobacterium phlei, M. smegmatis, $M$. fortuitum, $M$. gordonae, $M$. kansasi, $M$. bovis, $M$. tuberculosis and $M$. intracellulare were adapted to grow in an anaerobic environment. Concomitant with the transition to anaerobic growth was loss of acid-fastness, loss or modification of colonial pigmentation, and loss of ability to grow on a malachite green-containing medium. The mycobacteria grown anaerobically produced acid from a greater range of carbohydrates than aerobically grown cultures, lost iron-uptake activity, and showed a reduction of urease, catalase and nitratase activity. Back adaption of mycobacteria from an anaerobic to an aerobic environment resulted in the acquisition of acid-fastness, pigmentation, and other characteristics used in the taxonomy of mycobacteria. These results suggest that mycobacterial cultures, if grown in an anaerobic environment, may be erroneously identified in clinical laboratories.
\end{abstract}

\section{Introduction}

Mycobacteria are considered to be obligate aerobes that can grow in oxygen tensions that range from atmospheric to microaerophilic (Jenkins, et al., 1982; Moore and James, 1982). Research and clinical laboratories routinely observe that mycobacteria in broth cultures will clump, sink to the bottom of the tube and continue to grow in the pellet where oxygen levels would be low because of oxygen consumption by the cytochrome system and lactate oxidase (Ratledge, 1976). The report of survival of Mycobacterium tuberculosis in anaerobic conditions (Wayne and Lin, 1982) and the demonstration of ferredoxin in $M$. smegmatis (Imai et al., 1983) gave impetus to our investigation of anaerobic growth of mycobacteria.

Laboratory strains of mycobacteria were adapted to grow anaerobically and their physiological activities compared with those of strains grown aerobically. We describe procedures for the adaption of mycobacteria to different oxygen tensions and record the unique phenotypic characteristics of mycobacteria grown anaerobically.

\section{Materials and methods}

\section{Bacteria}

Nine strains of mycobacteria were used: $M$. phlei

\footnotetext{
* Requests for reprints should be addressed to L. L. Barton. Received 7 Aug. 1984; revised version accepted 30 Jun. 1985.
}

ATCC $354, M$. smegmatis ATCC $14468, M$. fortuitum TMC 1529, $M$. gordonae TMC $1318, M$. kansasi TMC 1201, $M$. intracellulare TMC 1403, M. tuberculosis H37Ra TMC 201, $M$. bovis BCG Pasteur TMC 1011 and $M$. phlei HMS, a smooth strain supplied by Dr J. Hanks, Harvard Medical School. TMC strains were from the Trudeau Mycobacterial Culture Collection, obtained from the National Jewish Hospital and Research Center, Denver, CO, USA.

\section{Media}

M. phlei and $M$. smegmatis were maintained on Trypticase Agar (Baltimore Biological Laboratories, Baltimore, MD) slants containing glycerol $0.2 \%$. All other strains were maintained on Lowenstein Jensen slants containing malachite green (Difco) $0.025 \%$. For routine maintenance in anaerobic conditions, a glycerol-trypticase medium consisting of glycerol $0.2 \%$, Tween $800.1 \%$, trypticase (BBL) $0.8 \%$ and sodium thioglycollate $0.05 \%$ was used. A $1-\mathrm{ml}$ inoculum from an actively growing culture was added to $10 \mathrm{ml}$ of freshly steamed medium in a $16 \mathrm{~mm} \times 125 \mathrm{~mm}$ tube. Inoculated tubes were placed in anaerobic jars containing Gas Pak $\mathrm{H}_{2}+\mathrm{CO}_{2}$ generators (Becton Dickinson, Cockeysville, MD).

\section{Adaptation of cultures}

The nine strains of mycobacteria were adapted to anaerobic growth by inoculation into Thioglycollate Broth without dextrose (Difco) supplemented with glycerol $0.2 \%$ and Tween $800 \cdot 1 \%$. After 9-12 serial transfers from the bottom of the micro-aerophilic growth zone, dense growth occurred throughout the bottom half of the tube. Transfers were made at approximately 4-week 
intervals. Following the eighth transfer, the tubes were incubated in anaerobic jars with a $\mathrm{H}_{2}+\mathrm{CO}_{2}$ atmosphere to eliminate the possibility that cultures could be growing in micro-aerophilic regions of the medium and cells sinking into the anaerobic zone.

To adapt the anaerobically growing mycobacteria to aerobic conditions, the cultures were inoculated into thioglycollate broth containing glycerol and Tween 80 and incubated in atmospheric conditions. After 4 weeks a subculture was made of growth from the uppermost level of the tube. After 8-10 serial transfers the organisms were transferred to Lowenstein Jensen slants and their physiological characteristics determined.

\section{Cultural Characters}

Colonial morphology and pigmentation. Colonial characteristics were recorded after growth for 3 weeks on a medium consisting of Nutrient Agar (Difco) 2.3\%, Yeast Extract (Difco) $0.4 \%$ and glycerol $0.2 \%$. Scotochromogenicity and photochromogenicity were determined according to the procedures recommended by Runyon et al. (1980). After inoculation the strains of mycobacteria were incubated in ambient conditions or in anaerobic jars containing $\mathrm{H}_{2}+\mathrm{CO}_{2}$ generators.

Growth characteristics. Strains were divided into rapidly or slowly growing groups on the basis of visible growth after incubation for 3 days in thioglycollate broth containing glycerol and Tween 80 . Sensitivity to malachite green $0.025 \%$ was revealed by failure of growth on Lowenstein Jensen slants after incubation for 28 days. Growth of rapidly growing mycobacteria in the presence of $\mathrm{NaCl} 5 \%$ was determined after incubation for 10 days in Kirchner's broth containing $\mathrm{NaCl} 5 \%$ (Hedgecock and Costello, 1962). Growth at $25^{\circ} \mathrm{C}, 33^{\circ} \mathrm{C}, 45^{\circ} \mathrm{C}$ and $54^{\circ} \mathrm{C}$ was determined with the glycerol-trypticase medium incubated for 10 days for rapidly growing and 6 weeks for slowly growing strains. Each of these characteristics was determined for the nine strains cultivated in aerobic and anaerobic conditions.

\section{Biochemical tests}

Preparation of cell suspensions. Aerobic and anaerobic cultures of mycobacteria were grown in a medium consisting of glycerol $0.2 \%$, Nutrient Broth (Difco) $0.8 \%$, Yeast Extract (Difco) $0.4 \%$, and Tween $800.1 \%$. Cells were collected by centrifugation, washed twice with 0.02 M phosphate buffer, $\mathrm{pH} 7 \cdot 0$, containing $\mathrm{NaCl} 0.8 \%$, and resuspended in phosphate buffered saline to an optical density of 0.4 at $540 \mathrm{~nm}$. For each test an inoculum of 0.1 $\mathrm{ml}$ was used.

Catalase. The semiquantitative catalase test described by Runyon et al. (1980) was used on strains grown for 2 weeks on Dubos' agar enriched with asparagine (Difco) $0.5 \%$. The spot test for catalase was performed by adding $\mathrm{H}_{2} \mathrm{O}_{2}$ to a colony from Dubos' agar (Finegold, et al., 1978).

Iron uptake. Rapidly growing mycobacteria were inoculated on to slopes of nutrient agar $2 \cdot 3 \%$, yeast extract $0.4 \%$, and glycerol $0.2 \%$. When growth was visible, ferric ammonium citrate $20 \%$ was overlaid on the slant. The appearance of rusty brown colonies after incubation for a further 21 days indicated a positive result (Wayne and Doubek, 1968).

Niacin test. Niacin production was detected in strains grown for 6 weeks on Dubos' agar enriched with asparagine $0.5 \%$ by the spot-plate test (Runyon, et al., 1959).

Nitrate reduction. The colorimetric tube test for nitrite production (Vestal, 1973) was used to test strains grown on nutrient agar $2.3 \%$, yeast extract $0.4 \%$, and glycerol $0.2 \%$. Rapidly growing strains were incubated for 1 week and slowly growing strains for 2 weeks before cells were tested for nitrate reduction.

Tween hydrolysis. Hydrolysis of Tween 80 was detected according to the procedure of Wayne, et al. (1964).

Urease. The procedure employed for hydrolysis of urea was a modification (Runyon et al., 1980) of the method of Toda et al. (1960).

Acid production from carbohydrates. Cystine Tryptic Broth (Difco) was used as the nutrient base for the evaluation of carbohydrate metabolism. After addition of carbohydrate $0.5 \%$ and $0.1 \mathrm{ml}$ of the OD 0.4 suspension, tubes were incubated in aerobic or anaerobic conditions for 10 days. Acid production in aerobic cultures was evaluated in the aerobic zone of the medium.

\section{Results}

The adaptation of mycobacteria to anaerobic growth produced cultures with characteristics distinct from their aerobic counterparts. Colonies formed in anaerobic conditions were smooth, moist and colourless. Most significant was the loss of acidfastness by cells grown anaerobically and stained by the Ziehl-Neelsen method. They would not grow in the presence of malachite green. Anaerobic cultures did not display unique cell morphologies and were characteristically composed of gram-positive rods. In broth culture, mycobacteria grew anaerobically without cell aggregation. Transition from aerobic to anaerobic or anaerobic to aerobic metablism was through an obligate microaerophilic state. Considerable sensitivity to oxygen was displayed by the mycobacteria gown anaerobically and inoculation of aerobic media resulted in cell death. With adaptation of anaerobic cultures to aerobic growth, the colonies became rough and regained pigmentation and cells displayed acid-fastness and the ability to grow in the presence of malachite green.

The temperature range in which growth occurred was the same for anaerobic and aerobic cultures of all the strains. Those strains that grew in the presence of $\mathrm{NaCl} 5 \%$ in aerobic conditions also grew anaerobically with the exception of $M$. fortuitum. Strains that failed to grow in the presence of $\mathrm{NaCl} 5 \%$ in aerobic conditions also failed to grow 
Table I. Physiological characteristics of rapidly growing mycobacteria

\begin{tabular}{|c|c|c|c|c|c|c|c|c|}
\hline \multirow[b]{2}{*}{ Character } & \multicolumn{2}{|c|}{ M. phlei 354} & \multicolumn{2}{|c|}{ M. phlei HMS } & \multicolumn{2}{|c|}{ M. smegmatis } & \multicolumn{2}{|c|}{ M. fortuitum } \\
\hline & aerobic & anaerobic & aerobic & anaerobic & aerobic & anaerobic & aerobic & anaerobic \\
\hline Pigmentation* & $\mathrm{S}$ & $\mathrm{N}$ & $\mathbf{S}$ & $\mathrm{N}$ & $\mathbf{N}$ & $\mathrm{N}$ & $\mathrm{N}$ & $\mathbf{N}$ \\
\hline $\begin{array}{l}\text { Growth on } \\
\text { Lowenstein Jensen }\end{array}$ & & & & & & & & \\
\hline plus malachite green & + & - & + & - & + & - & + & - \\
\hline Catalase production & + & - & + & - & + & - & + & - \\
\hline Nitrate reduction & + & - & + & + & + & - & + & - \\
\hline Urease production & + & - & + & - & + & - & + & - \\
\hline Tween hydrolysis & + & + & + & - & + & - & + & - \\
\hline Iron uptake & + & - & + & - & + & - & - & - \\
\hline
\end{tabular}

* $\mathrm{S}=$ Scotochromogen, $\mathrm{P}=$ photochromogen, $\mathrm{N}=$ no pigment.

$+=$ Positive result in test; $-=$ negative result in test.

Table II. Physiological characteristics of slowly growing mycobacteria

\begin{tabular}{|c|c|c|c|c|c|c|c|c|c|c|}
\hline \multirow[b]{2}{*}{ Character } & \multicolumn{2}{|c|}{ M. gordonae } & \multicolumn{2}{|c|}{ M. kansasi } & \multicolumn{2}{|c|}{$M$. intracellulare } & \multicolumn{2}{|c|}{ M. tuberculosis } & \multicolumn{2}{|c|}{ M. bovis } \\
\hline & aerobic & anaerobic & aerobic & anaerobic & aerobic & anaerobic & aerobic & anaerobic & aerobic & anaerobic \\
\hline Pigmentation & S & $N$ & $\mathbf{P}$ & $\mathbf{N}$ & $\mathrm{N}$ & $\mathbf{N}$ & $\mathbf{N}$ & $\mathrm{N}$ & $N$ & $N$ \\
\hline $\begin{array}{l}\text { Growth on } \\
\text { Lowenstein Jensen }\end{array}$ & & & & & & & & & & \\
\hline plus malachite green & + & - & + & - & + & - & + & - & + & - \\
\hline Catalase production & + & - & + & - & - & - & - & - & - & - \\
\hline Nitrate reduction & - & + & + & - & - & + & + & - & - & - \\
\hline Urease production & - & + & - & + & + & + & + & + & + & - \\
\hline Tween hydrolysis & + & - & + & + & - & - & - & - & - & - \\
\hline
\end{tabular}

See footnote to table I.

Table III. Acid production from carbohydrates by rapidly growing mycobacteria

\begin{tabular}{|c|c|c|c|c|c|c|c|c|}
\hline \multirow[b]{3}{*}{ Carbohydrate } & \multicolumn{8}{|c|}{ Acid production by } \\
\hline & \multicolumn{2}{|c|}{ M. phlei 354} & \multicolumn{2}{|c|}{ M. phlei HMS } & \multicolumn{2}{|c|}{ M. smegmatis } & \multicolumn{2}{|c|}{$M$. fortuitum } \\
\hline & aerobic & anaerobic & aerobic & anaerobic & aerobic & anaerobic & aerobic & anaerobic \\
\hline Arabinose & + & + & - & + & + & + & + & + \\
\hline Dulcitol & + & + & - & + & + & + & - & + \\
\hline Fructose & - & + & + & + & + & + & - & - \\
\hline Galactose & - & + & - & + & - & + & - & - \\
\hline Glucose & + & + & + & + & + & + & - & - \\
\hline Glycerol & - & + & - & + & + & + & - & + \\
\hline Inositol & - & + & - & + & + & + & - & + \\
\hline Mannitol & - & + & - & + & + & + & - & + \\
\hline Mannose & - & + & - & + & + & + & + & + \\
\hline Rhamnose & + & - & - & - & + & - & - & - \\
\hline Sorbitol & + & + & - & + & + & + & - & + \\
\hline Trehalose & + & + & - & + & - & + & + & + \\
\hline Xylose & + & + & + & + & + & + & - & - \\
\hline
\end{tabular}

$+=$ Acid production associated with growth, as determined by phenyl red $p \mathrm{H}$ indicator; $-=$ No acid production. 
anaerobically. All strains of mycobacteria grew aerobically and anaerobically in Kirchner's broth without $\mathrm{NaCl}$.

Other physiological characteristics of the strains are shown in tables I and II. Carotenoids were produced by $M$. phlei, $M$. gordonae and $M$. kansasi in aerobic conditions only. Anaerobic cultures of both slowly and rapidly growing mycobacteria were sensitive to malachite green. The spot test for catalase was positive with all the strains grown in aerobic and anaerobic conditions, but the semiquantitative catalase test was negative with strains grown anaerobically. With the exception of $M$. phle $i$ HMS, strains that reduced nitrate in aerobic conditions were unable to do so anaerobically. Urease activity was present in aerobic cultures of the four rapidly growing strains and $M$. bovis but not in anaerobic cultures. Of the slowly growing strains, $M$. tuberculosis and $M$. intracellulare showed urease activity in aerobic and anaerobic conditions, but only the anaerobic cultures of $M$. gordonae and $M$. kansasi were urease-positive. The ability to hydrolyse Tween 80 was lost with anaerobic adaptation of $M$. phlei HMS, M. smegmatis, $M$. fortuitum, and $M$. gordonae but not $M$. phlei 354 or $M$. kansasi. Iron uptake was observed only with aerobic cultivation of $M$. phlei and M. smegmatis.

All the rapidly growing mycobacteria grew on the medium used to test for the production of acid from carbohydrates. $M$. fortuitum produced acid from fewer carbohydrates than either $M$. phlei or $M$. smegmatis and a marked difference in metabolism was seen between aerobic and anaerobic cultivation of $M$. phlei, M. smegmatis, and M. fortuitum (table III).

\section{Discussion}

Mycobacteria were adapted in the laboratory to grow in anaerobic conditions and the resulting cultures displayed characteristics significantly different from those of their aerobic counterparts. Anaerobic growth of mycobacteria was achieved only through a stepwise decrease in oxygen tension thereby distinguishing this growth response from that observed with facultative anaerobes. The difficulty in attaining growth of mycobacteria and the loss of phenotypic characteristics of the cells indicates that the anaerobic growth potential of the mycobacteria would not be revealed in normal circumstances.

The growth obtained in anaerobic conditions was indeed that of mycobacteria, as demonstrated by the return of phenotypic characters typically associated with mycobacteria when the strains were adapted to grow again in aerobic culture. In general, physiological characteristics of salt tolerance, temperatures for growth, and resolution into rapidly and slowly growing mycobacteria, were shared by both aerobic and anaerobic cultures.

Oxygen tension has a marked effect on the metabolism of mycobacteria. Growth of $M$. tuberculosis $\mathrm{H} 37 \mathrm{Rv}$ in oxygen-limited conditions results in the production of aldolase type I whereas growth in oxygen-rich conditions yields aldolase type II (Bai et al., 1975). Lactate oxygenase activity is directly influenced by oxygen concentration (Ratledge, 1976) and lactic acid could be the acidogenic product of glycolysis (Szymona, et al., 1967). Although no direct evidence is available for linking oxygen concentration with the chromophobic character of the tubercle bacilli (Nyka, 1967), it would be important to determine if this unusual response to the acid-fast stain is attributable to altered oxygen levels in vivo. The hypothesis that virulent mycobacteria tolerate lower oxygen tensions than avirulent strains (Rypka, 1958) merits reexamination.

The phenotypic changes concomitant with adaption of mycobacteria to anaerobic growth could have a significant impact on identification of mycobacteria isolated from clinical specimens. While it is possible that mycobacteria may be isolated in anaerobic conditions, and subsequent characterisation could lead to an erroneous identification, it should be noted that anaerobic cultures of specimens are incubated anaerobically usually for $48 \mathrm{~h}$, and not longer than 7 days. It is unlikely that mycobacteria would grow in these anaerobic cultures of clinical specimens because the time of incubation would be too short, especially for the slowly growing strains. Future studies of cultivation of mycobacteria in oxygen-deficient environments will enhance our understanding of this most important group of bacteria.

The research was supported in part by a grant to LLB from the Faculty Research Allocations of the University of New Mexico. 


\section{REFERENCES}

Bai N J, Pai M R, Murthy P S, Venkitasubramanian T A 1975 Pathways of carbohydrate metabolism in Mycobacterium tuberculosis H37RV. Canadian Journal of Microbiology 21:1688-1691.

Finegold S M, Martin W J, Scott E G (eds) 1978 Bailey and Scott's Diagnostic Microbiology, 5th edn. C. V. Mosby, St Louis, p 482 .

Hedgecock L W, Costello R L 1962 Utilization of nitrate by pathogenic and saprophytic mycobacteria. Journal of Bacteriology 84:195-205.

Imai T, Matsumoto T, Ohta S, Ohmori D, Suzuki K, Tanaka J, Tsukioka M, Tobari J 1983 Isolation and characterization of a ferredoxin from Mycobacterium smegmatis Takeo. Biochimica et Biophysica Acta 743:91-97.

Jenkins P A, Pattyn S R. Portaels F 1982 Diagnostic bacteriology. In: Ratledge C, Stanford J (eds) Biology of the mycobacteria, vol. 1. Academic Press, New York, pp 441 470 .

Moore D F, James A M 1982 Growth studies on Mycobacterium BCG: Oxygen preference. Microbios 35:151-159.

Nyka W 1967 Method for staining both acid-fast and chromophobic tubercle bacilli with carbolfuchsin. Journal of Bacteriology 93: 1458-1460.

Ratledge C 1976 The physiology of the mycobacteria. Advances in Microbial Physiology 13:115-244.

Runyon E H, Karlson A G, Kubica G P, Wayne L G 1980 Mycobacterium. In: Lennette, E $H$ et al. (eds) Manual of clinical microbiology, 3rd edn. American Society for Microbiology, Washington DC, pp 165-171.

Runyon E H, Selin M J, Harris H W 1959 Distinguishing mycobacteria by the niacin test. American Review of Tuberculosis on Pulmonary Diseases. 79:663-665.

Rypka E W 1958 Growth of avirulent and virulent tubercle bacilli. Ph.D. Dissertation, Stanford University, Palo Alto, CA.

Szymona O, Pinkiewicz H, Szumilo T 1967 Comparative studies on some enzymes of atypical and other mycobacteria. Acta Microbiologica Polonica 16:101-114.

Toda T, Hagihara Y, Takeya K 1961 A simple urease test for the classification of mycobacteria. American Review of Respiratory Diseases 83:757-761.

Vestal A L 1973 Procedures for the isolation and identification of mycobacteria. 73-8230. Center for Disease Control, Atlanta, GA.

Wayne L G, Doubek J R 1968 Diagnostic key to mycobacteria encountered in clinical laboratories. Applied Microbiology 16:925-931.

Wayne L G, Doubek J R, Russell R L 1964 Classification and identification of mycobacteria. I. Tests employing Tween 80 as substrate. American Review of Respiratory Diseases 90:588-597.

Wayne L G, Lin K-Y 1982 Glyoxylate metabolism and adaptation of Mycobacterium tuberculosis to survival under anaerobic conditions. Infection and Immunity 37:1042 1049. 